\title{
Daniel Fabre (1947-2016)
}

Josiane Bru

\section{(2) OpenEdition}

Journals

Édition électronique

URL : https://journals.openedition.org/clo/2547

DOI : $10.4000 /$ clo. 2547

ISSN : 2266-1816

Éditeur

INALCO

\section{Édition imprimée}

Date de publication : 1 janvier 2016

ISBN : 9782858312337

ISSN : 0396-891X

\section{Référence électronique}

Josiane Bru, «Daniel Fabre (1947-2016) », Cahiers de littérature orale [En ligne], 79 | 2016, mis en ligne le 20 décembre 2016, consulté le 30 juin 2021. URL : http://journals.openedition.org/clo/2547 ; DOI : https://doi.org/10.4000/clo.2547

\section{(c) $($ ) 8}

Cahiers de littérature orale est mis à disposition selon les termes de la Licence Creative Commons Attribution - Pas d'Utilisation Commerciale 4.0 International. 


\section{Daniel Fabre (1947-2016)}

Josiane BRU

« Notre intérêt pour cette portion des biens symboliques que l'on nomme littérature orale date de 1966 » écrivaient Daniel Fabre et Jacques Lacroix dans le volumineux ouvrage en deux volumes issu, au début des années soixante-dix, de leur thèse sur le Conte populaire dans la Haute-vallée de l'Aude.

Cet intérêt, étendu à la question du symbolique en général qui lui fait opérer un retour périodique pour interroger à la fois son propre parcours et jusqu'aux fondements de sa discipline, ont nourri l'anthropologie de Daniel Fabre dès les premières années de son enseignement, à partir de 1974, à l'université et dans le cadre du pôle toulousain de l'EHESS. Renvoyant le lecteur aux nombreux hommages qui lui ont été rendus, nous revenons ici sur cette période maintenant occultée par l'ampleur de ses champs de recherche et d'intervention ultérieurs, en particulier par son rôle moteur à la Mission du patrimoine ethnologique et dans l'institutionnalisation du patrimoine culturel immatériel.

Partant du Pays de Sault, un plateau isolé des Pyrénées audoises, l'enquête initiale s'étend très vite à l'ensemble des savoirs et pratiques de la société rurale occitane. Les deux jeunes ethnologues qui l'animent dès les premières années revendiquent d'entrée leur appartenance à la société qu'ils étudient et affirment leur désir de la conduire dans la langue naturelle du pays, la leur. Ils ont plus largement l'ambition d'impulser, face au regard (et au non-regard) porté par les intellectuels français sur la société paysanne, le projet d'une anthropologie autochtone, « décolonisée », envisageant de l'intérieur, en toute conscience et au moment où elles se dissolvent dans la société industrielle et citadine, les racines revendiquées par les minorités nationales en voie d'émergence.

Les maîtres, ou plutôt les aînés, sont eux aussi engagés dans des travaux d'inventaire et d'analyse de la culture occitane et de son histoire : inventaire linguistique pour le dialectologue Jean Séguy, inventaire philosophique, littéraire et historique pour le poète René Nelli, spécialiste du catharisme et de la poétique 
des troubadours. Figure tutélaire de la vie intellectuelle intense de Carcassonne à cette époque, il animait Folklore, Revue d'ethnographie méridionale, qui fédérait plusieurs générations d'intellectuels et d'artistes défendant la culture d'Oc dans toutes ses dimensions. Les jeunes chercheurs en sciences sociales qui suivaient leurs enseignements à l'université de Toulouse constitueront les forces vives du Centre d'Anthropologie des Sociétés Rurales (EHESS et CNRS) créé en 1978, par Daniel Fabre avec l'archéologue Jean Guilaine sur une proposition de Jacques Le Goff. La plupart de ses membres sont des enseignants de lycée ou collège engagés dans des projets de thèses sur le Pays de Sault. Glissant progressivement vers des terrains et des thématiques plus larges, ils intègreront ensuite les organismes d'enseignement supérieur et de recherche. Devenus des familiers de leurs informateurs dont ils interrogeaient les discours, croyances et autres « façons de dire [et] façons de faire » le groupe d'ethnologues réuni autour de D. Fabre, fut désigné un temps comme « école de Toulouse ».

La fête est un des lieux où s'expriment avec évidence les héritages du passé, de celle qui met en scène un ours ravisseur de filles, humanisé par le rasage final qui le rend à la société villageoise jusqu'aux carnavals des villages languedociens et leurs animaux totémiques. Carnaval étudié, carnaval vécu et joué à Carcassonne où l'ethnologue endosse le rôle dans le temps même où il interroge le rapport des jeunes à la mort à travers leurs jeux rituels et leurs facéties qui passent par « la voix des masques ». Le récit oral, collectif dans son élaboration et sa transmission, en est un autre. La moitié du corpus des 150 contes recueillis dans divers villages entre 1970 et 1973 furent publiés dans la Tradition orale du conte occitan. Les commentaires montrent l'intérêt des auteurs pour les outils d'analyse de ces corpus et particulièrement pour le catalogue du Conte populaire français dont, des années plus tard, D. Fabre accueillera le chantier à Toulouse. Au fil des années, il puisera dans ces récits, pris dans le contexte de la société qui les leur a transmis, les éléments d'une longue investigation sur l'invisible initiation des garçons.

Performance réitérée et récit-modèle dans lequel s'opère le passage de l'enfance à l'âge adulte, le conte est au même titre que les rites et autres pratiques coutumières un des points d'ancrage de son interrogation sur la construction sociale des garçons et leur accès à la virilité. Plusieurs articles en témoignent où les sources de la réflexion - écrites, orales ou vécues - se fondent dans l'écriture du chercheur tout comme dans le discours fluide qu'il déploie dans ses séminaires en tant que chargé de conférence à l'EHESS où il est nommé directeur d'études en 1989. Il y parle comme un conteur, ménageant ses effets, ouvrant parfois de longues et saisissantes parenthèses qui laissent ses auditeurs le stylo en l'air et bouche bée. Les thèmes s'y succèdent comme autant de récits d'investigation sur les limites - celles du sauvage et du domestique, du monde des vivants et du monde des 
morts - et sur leur transgression qui, comme dans les contes, permet à chacun d'aller vers son destin ou d'en mourir. Jean de l'Ours, qui tire sa force de son incertaine naissance pour parcourir le monde et y faire sa place et va jusqu'au bout de sa quête malgré la trahison de ses compagnons fut le premier des héros interrogés. Suivirent Jean sans Peur, que le frisson provoqué par des battements d'ailes décide enfin à se marier, Jean le Sot - anti-héros carnavalesque, ce raté de l'initiation qui ne comprendra jamais les métaphores du langage des oiseaux - et tous les personnages dont l'histoire réelle ou fictive raconte l'itinéraire initiatique.

Émergeant progressivement, d'abord comme signe d'appartenance sur le bétail puis dans les maisons paysannes où parallèlement à l'imprimé - almanach ou livre de magie - l'écriture devient progressivement le fil conducteur qui relie les deux grandes périodes de l'œuvre. Daniel Fabre en scrute/explore les diverses formes, de l'autobiographie qui fait l'objet des séminaires dès 1987 aux écritures ordinaires, de l'autographie à l'écriture romanesque chez les écrivains qui tentent d'en saisir en eux-mêmes le douloureux processus d'engendrement. En lien avec la création d'un nouveau centre de recherches, parisien cette fois - le Laboratoire d'Anthropologie et d'Histoire des Institutions de la Culture - la littérature écrite et la question du patrimoine immatériel ont constitué le centre de la seconde partie d'une œuvre ponctuée, comme la première, de retours sur l'anthropologie comme discipline et dans laquelle tout se tient étroitement lié.

« Au fond, je n'ai jamais écrit que sur moi-même » aurait-il confié à des fidèles de ses séminaires en même temps que son désir de revenir physiquement sur ses premiers terrains. C'est sans doute cette quête de soi, de sa propre construction entre ce qui parle au plus profond et les aspirations singulières, qui donne sa force et sa cohérence à une œuvre dans laquelle tout est annoncé dès le départ, mais que le temps ne lui a pas permis d'accomplir jusqu'au bout. Juste de revisiter de mémoire, ces dernières années et dans quelques articles, ses territoires de la jeunesse.

\section{Bibliographie}

Pour plus ample information on consultera les sites de l'ethnopôle GARAE www. garae.fr/ et du laboratoire IIAC/LAHÏC http://www.iiac.cnrs.fr/rubrique69. $\underline{\mathrm{html}}$

FABre, Daniel et LACroix, Jacques, 1970, « Una contairina populara audenca », Obradors, Montpellier : Centre d'Études Occitanes, Université Paul Valéry. 
10 Des vies extraordinaires : les territoires du récit $-\mathrm{n}^{\circ} 79$

Fabre, Daniel et Lacroix, Jacques, 1973 et 1975, la Tradition orale du conte occitan. Les Pyrénées audoises, Paris : PUF, 2 vol.

Fabre, Daniel et Camberoque, Charles, 1977, la Fête en Languedoc, regards sur le carnaval aujourd'bui, Toulouse : Privat.

FAbre, Daniel et Ariès Philippe, 1982, « Du messager des âmes au spirite » in la Mort Aujourd'bui, Marseille : Rivages, p. 95-109.

Fabre, Daniel, 1982, « Pierre Pous, conteur du Pays de Sault », Cabiers de littérature orale, $\mathrm{n}^{\circ} 11, \mathrm{p} .146-149$.

Fabre, Daniel et Blanc, Dominique, 1982, le Brigand de Cavanac; le fait divers, le roman, l’histoire, Lagrasse : Verdier. Nouvelle éd. complétée, 2015.

FAbre, Daniel, 1985, « Le livre et sa magie », in Pratiques de la lecture, Marseille : Rivages, p. 181-206.

FABRE, Daniel, 1986, « La voie des oiseaux, sur quelques récits d'apprentissage », l'Homme, revue française d'anthropologie, no 98, p. 7-34, http://www.persee. fr/doc/hom_0439-4216_1986_num_26_99_368712.

FABRE, Daniel, 1986, « Le garçon enceint », Cahiers de littérature orale, n²0, p. 15-38.

FABRE, Daniel (dir.), 1987, Études rurales, nº 105-106: Le retour des morts.

FABRE, Daniel (dir.), 1991, Ethnologie française, t. 21, nº 4 : Apprentissages, hommage à Yvonne Verdier.

Fabre, Daniel (dir. avec G. Althabe et G. Lenclud), 1992, Vers une ethnologie du présent, Paris, MSH.

FABRE, Daniel (dir.), 1993, Écritures ordinaires, Paris, P.O.L.

FABre, Daniel, 1996, « Le symbolique, brève histoire d'un objet », in une École pour les sciences sociales, Paris : Cerf et Éd. de l'EHESS, p. 229-250.

FABre, Daniel (dir. avec Alban Bensa), 2001, Une Histoire à soi, Paris, MSH. 
Fabre, Daniel (dir.), 2009, Gradhiva, n 9 : Arts de l'enfance, enfances de l'art.

FABRE, Daniel, 2009, « Fondu au noir », l'Homme, revue française d'anthropologie, $\mathrm{n}^{\circ} 191$, p. 27-36.

FABre, Daniel (dir. avec J. Jamin et M. Massenzio), 2010, l'Homme, revue française d'anthropologie, no 195-196: Auto-biographie, ethno-biographie.

Fabre, Daniel, 2014, « Marcel Proust en mal de mère. Une fiction du créateur », Gradhiva, n² 20, p. 48-83, https://gradhiva.revues.org/2831. 